\title{
Lennox-Gastaut Syndrome: An Update on Treatment
}

\author{
Lionel Carmant, Sharon Whiting
}

\begin{abstract}
Lennox-Gastaut syndrome (LGS) is a severe epileptic encephalopathy. Few current treatment options are effective in improving seizure control. This paper reviews the available treatments of LGS and discusses a new option in Canada, rufinamide. It is a wide spectrum anticonvulsant, approved in a number of countries for the treatment of LGS. In a randomized controlled trial in the LGS population, adjunctive rufinamide therapy has been shown to offer significantly greater reduction in total seizure frequency and tonic-atonic seizure frequency in comparison to placebo. Efficacy has been assessed over three years and appears to be sustained. Most adverse events were cognitive (e.g. somnolence) or gastrointestinal in nature and in many cases transient or mild. Based on the efficacy and safety data on rufinamide obtained to date, this medication will provide additional benefits to patients with LGS in Canada and is an important consideration for our patients in the adjunctive treatment setting.
\end{abstract}

RÉSUMÉ: Mise-à -jour sur le traitement de syndrome de Lennox-Gastaut. Le syndrome de Lennox-Gastaut (SLG) est une encéphalopathie épileptique sévère. Peu des options thérapeutiques actuelles sont efficaces pour améliorer le contrôle des crises. Dans cet article, nous revoyons les traitements du SLG qui sont disponibles et nous discutons d'une nouvelle option thérapeutique disponible au Canada, le rufinamide. Il s'agit d'un anticonvulsivant à large spectre qui a été approuvé dans certains pays pour traiter le SLG. Un essai clinique randomisé chez des patients atteints du SLG a démontré qu'un traitement d'appoint par le rufinamide entraînait une diminution significativement supérieure de la fréquence totale des crises et de la fréquence des crises toniques-atoniques comparé au placebo. Son efficacité a été évaluée pendant 3 ans et semble persister. La plupart des incidents thérapeutiques étaient de nature cognitive (e.g. la somnolence) ou gastrointestinaux et ils étaient transitoires ou légers chez plusieurs patients. Les données actuelles sur l'efficacité et la sécurité du rufinamide indiquent que cette médication apportera des bénéfices additionnels aux patients atteints de SLG au Canada et constitue un traitement d'appoint intéressant à considérer chez nos patients.

Can J Neurol Sci. 2012; 39: 702-711

\section{DEFINITION AND EPIDEMIOLOGY}

Lennox-Gastaut syndrome (LGS) is a rare but devastating childhood epilepsy syndrome. The term "Lennox-Gastaut syndrome" was first used in the literature in 1969. ${ }^{1}$ The definition in use today was originally published in 1989 by The International League Against Epilepsy (ILAE) in their "Proposal for Revised Classification of Epilepsy and Epileptic Syndromes":2

"Most common seizure types include tonic-axial, atonic, and absence seizures, but myoclonic, generalized tonic-clonic or partial seizures are frequently associated with LGS. Frequency of seizures and status epilepticus (stuporous states with myoclonic, tonic, and atonic seizures) is high. The electroencephalogram (EEG) typically shows abnormal background activity, slow spike-and-waves less than $3 \mathrm{~Hz}$ and often multifocal discharges. Bursts of fast rhythms ( 10 Hz) appear during sleep. There is typically mental retardation."

The ILAE "revised terminology and concepts for organization of seizures," characterizes LGS as an electroclinical syndrome of childhood onset. ${ }^{3}$ Lennox-Gastaut syndrome has been reported to account for anywhere from one to ten percent of all cases of childhood epilepsy ${ }^{4,5}$ and is more prevalent in males than females. ${ }^{4,6,7}$ This broad incidence range seems to be the result of differing interpretation of the classic criteria to diagnose LGS. ${ }^{7}$ A Nova Scotia population-based study of children with new-onset epilepsy (i.e. incident cases) determined an incidence of $12 \%$ for symptomatic generalized epilepsies (SGEs), however only four percent of children with SGEs were diagnosed with LGS. ${ }^{8,9}$ Therefore the incidence of LGS in all new-onset epilepsies was only $0.6 \%$. In another population based study, LGS represented four percent of all epilepsy syndromes and its prevalence was estimated at $0.26 / 1000$ ten year-old children. ${ }^{10}$ Sixty to eighty percent of patients with LGS experience seizures which are refractory to treatment. ${ }^{11}$ Patients with LGS are often

From the Université de Montréal (LC), Hôpital Sainte-Justine, Montreal, Quebec; Faculty of Medicine (SW), University of Ottawa, Children's Hospital of Eastern Ontario, Ottawa, Ontario, Canada.

Received December 9, 2011. Final Revisions Submitted June 14, 2012. Correspondence to: Lionel Carmant, Université de Montréal, Hôpital Sainte-Justine, Room 5421, 3175 Côte Ste-Catherine, Montréal (Québec) H3T 1C5, Canada. 
prescribed multiple seizure medications (AEDs), occasionally in combination with non-pharmacological treatment options such as the ketogenic diet, epilepsy surgery, or vagus nerve stimulation. ${ }^{12}$ Despite these intensive treatment regimens, many patients are typically experiencing more than 90 seizures a month. ${ }^{4}$ Morbidity is significant, ${ }^{10}$ as head injuries occur, with patients needing to wear helmets as a preventative measure ${ }^{13}$ some patients even become wheelchair bound as a result of violent drop attacks. ${ }^{14}$

Early diagnosis of LGS continues to be a major challenge for physicians managing patients with refractory generalized epilepsy syndromes depending on how strictly the diagnostic criteria are applied, particularly for seizure types and EEG. This might lead to unnecessary delays in the initiation of appropriate treatment and to a worse prognosis as discussed below.

\section{Clinical Characteristics}

The three core symptoms of LGS (as defined by the ILAE) of multiple seizure types, including the hallmark tonic seizures, abnormal EEG characterized by a slow spike-and-wave pattern at a frequency of $<3 \mathrm{~Hz}$, and mental retardation, ${ }^{2}$ have come to be known as the "LGS triad."

Seizure types. Tonic seizures are the hallmark of LGS, differentiating it from other generalized epilepsies, but they may appear only during sleep, emphasizing the need to perform sleep EEGs when the diagnosis is suspected. ${ }^{15}$ Lennox-Gastaut syndrome presents in children aged one to eight years-of-age, with the majority of cases emerging between three and five years-of-age. ${ }^{7}$ In a United States (US) study, $82 \%$ of children who had a diagnosis of LGS experienced their first seizure within their first year of life with $22.7 \%$ having experienced neonatal seizures and $39 \%$ infantile spasms. ${ }^{10}$ Early diagnosis can be challenging given the number of different ways LGS can initially present and the lag between the first seizure and display of the full classic triad, which may take several years to emerge. ${ }^{8}$ In a retrospective study, 38 of 103 patients referred to an epilepsy centre with LGS had been misdiagnosed. ${ }^{16}$ In terms of seizures, the "core seizures" of LGS (tonic, atonic, and atypical absence) may not be the first seizure type to appear in a patient. ${ }^{12}$ A patient may present with any one of a variety of seizure types, including partial, myoclonic, and generalized tonic-clonic. ${ }^{2}$ Some seizures can be difficult to detect in infants. Notably, drop attacks cannot occur until the patient has begun to sit or stand ${ }^{17}$ and tonic seizures can be missed as they may present only in sleep. ${ }^{15}$

EEG findings. The diagnostic hallmark of LGS is the slow spike-and-wave seen on the EEG, but even these changes may not be present at the onset. ${ }^{11}$ Even when the awake EEG is abnormal at onset, the sleep EEG may still be normal and only gradually becomes abnormal with the appearance of epileptic recruiting rhythms or paroxysmal fast activity as typical EEG finding. In a statistical analysis of 80 patients with LGS, Chevrie and colleagues found that only $12.5 \%$ of patients displayed slow spike-and-waves at the time of their first seizure. ${ }^{17}$ Furthermore, this EEG pattern is not a unique feature of LGS. ${ }^{12}$ Slow spikeand-waves can also be seen in focal epilepsy with secondary bilateral synchrony.

Cognitive functions. Patients may present initially with normal psychomotor development or global delay (with or without behavioural disorder). ${ }^{12}$ Chevrie and colleagues found that $30 \%$ of patients presented with psychomotor delay prior to onset of LGS, however psychomotor delay can often be underdiagnosed in infants so, in reality, this percentage may be higher. ${ }^{17}$

\section{ETIOLOGY}

Traditionally, classification of LGS includes symptomatic and cryptogenic/idiopathic cases or according to the new classification: genetic, structural/metabolic, and of unknown etiology. ${ }^{18}$ The presentations of LGS can help predict the etiology and are divided into three categories: those cases which are the result of a brain injury, those with a previous history of infantile spasms, and those which present in previously normally developing children.

For patients with LGS with brain injury, the insult/injury typically occurs either prenatally or within the first year of life and is bilateral, diffuse or multifocal in nature. ${ }^{7}$ In patients with no known etiology, the drop attacks will usually be the first seizure type. ${ }^{12}$ While a genetic basis for LGS has not yet been determined, a family history of epilepsy is reported in 3 to $30 \%$ of patients with LGS. ${ }^{7}$ Mutation of the SCN1A gene, coding for the sodium channel alpha 1 subunit, has been associated with epileptic encephalopathies. ${ }^{19,20}$ Although rare, patients with this mutation can develop LGS. It is important to identify SCN1A mutations because the associated seizures are known to respond best to drugs which bind to the GABA receptor. ${ }^{21}$ The incidence of a family history of epilepsy is higher in cryptogenic versus symptomatic patients with LGS. ${ }^{7}$ With respect to West syndrome, approximately 23 to $54 \%$ of children with this syndrome go on to develop LGS. ${ }^{22}$ The causes of West syndrome are similar to LGS in that it may either be the result of an insult or of a genetic abnormality including malformations of cortical development. Some have even proposed that West syndrome and LGS may be the result of similar processes manifesting at different ages.

The varied onset of LGS can play a major role in when the diagnosis of LGS is made, and challenge the treating clinician to determine at what point in the emerging syndrome continuum LGS-appropriate treatments should be introduced into patient management. For example, the American Academy of Neurology/American Epilepsy Society (AAN/AES) definition of LGS published in 2004 included mental retardation, multiple seizure types, and characteristic EEG, including slow spike-andwave pattern. ${ }^{23}$ This definition does not include specific seizure types (such as tonic seizures) or fast rhythms during sleep. This somewhat broad definition might lead to the inclusion of patients with Dravet syndrome, Doose syndrome, or even some focal cryptogenic epilepsies, especially of frontal lobe origin. ${ }^{24}$ Most of the diagnostic criteria for LGS can be seen in other epilepsy syndromes, which is why a high degree of clinical suspicion is important. ${ }^{8}$ Clinicians may adhere to a more or less strict definition of LGS, and thus may elect to initiate LGS-specific treatments at different time-points in patient care.

\section{MANAgEMENT OF LGS}

In day-to-day clinical practice de novo cases of LGS are relatively rare. The LGS triad provides guidance as to what is required for a classic diagnosis of LGS but there is some debate over which seizure types are required and whether fast rhythms 
during sleep are necessary. ${ }^{24}$ As discussed, at onset the first seizure type experienced may not be one of the classic seizure types of LGS (tonic, atonic, and atypical absence), the initial EEG may not be abnormal and the patient may not present with cognitive delay. ${ }^{12,17}$ It may take several years for all the diagnostic features of LGS to become evident. ${ }^{8}$ The particular LGS definition a clinician adheres to may determine whether the patient is diagnosed as having LGS or a non-specific epileptic encephalopathy. Faced with this clinical reality, a management strategy based on the seizure types experienced by the patient, and not the diagnosis, is frequently used in an attempt to gain seizure control and improve the patient's and the caregiver's quality of life.

\section{Goals of Therapy}

Given that only a minority of patients with LGS achieve seizure freedom, ${ }^{25,26}$ the main goal of pharmacotherapy is a reduction in the frequency and severity of seizures to improve quality of life. ${ }^{14,27}$ High frequency of seizures reduces the healthrelated quality of life for patients as well as caregivers. ${ }^{14} \mathrm{~A}$ reduction in seizure frequency can lead to greater alertness, improvements in behaviour and cognitive function, ${ }^{12}$ a reduction in injuries, less disruption of school, and less impact on social and family relationships. ${ }^{27}$ Seizure frequency should not be the only criterion used to measure success of treatment. Aggressively pursuing a reduction in seizure frequency can lead to debilitating side effects, which are associated with some AEDs. ${ }^{12}$ Patients are often on multiple seizure medications, and may also pursue non-pharmacological treatment options. The treatment of LGS requires a multidisciplinary team consisting of psychologists, physiotherapists, occupational therapists, social workers, care workers and educators. ${ }^{27}$

\section{Current Management Guidelines for Seizure Medications (AEDs)}

Prior to 1990 there were only six major seizure medications, these included: carbamazepine, phenobarbital, phenytoin, primidone, ethosuximide, and valproic acid. ${ }^{28}$ These older drugs have the advantage of broad familiarity, known efficacy, and long-term experience. However, the efficacy of these older drugs is not supported by the same randomized controlled trials that are required for newer seizure medications. While valproic acid is generally considered to be the first-line treatment for LGS, the highest level of evidence for efficacy of this drug in LGS is class IV evidence (uncontrolled studies, case series, case reports, or expert opinion) according to the American Academy of Neurology (AAN) criteria. ${ }^{28}$ A number of new treatments have undergone testing in LGS in the past several decades.

The most recent treatment guidelines available from the AAN/American Epilepsy Society (AES) and the National Institute for Clinical Excellence (NICE) are from 2004, however, a draft of new NICE guidelines is available. ${ }^{23,29}$ The 2004 AAN/AES publication investigated seven new treatments: gabapentin, lamotrigine, topiramate, tiagabine, oxcarbazepine, levetiracetam and zonisamide. ${ }^{23}$ This publication recommended, in addition to valproic acid, topiramate and lamotrigine for treatment of drop attacks associated with LGS. The most recent NICE guidelines draft also recommend valproic acid as first-line treatment, lamotrigine as adjunctive second-line treatment and topiramate and rufinamide as third-line treatment. ${ }^{29}$ In 2005, a group of pediatric epilepsy specialists in the US were surveyed for their opinion on the treatment of LGS and other epilepsy disorders. ${ }^{30}$ The majority recommended valproic acid as firstline treatment, while lamotrigine and topiramate were also deemed appropriate first-line options. Second-line agents were: zonisamide, levetiracetam, felbamate, and the ketogenic diet. The majority also recommended attempting two monotherapies before initiating polytherapy. While, polytherapy increases the potential for adverse drug reactions, ${ }^{12}$ our clinical experience is that there is little or no advantage of using two monotherapies, as most patients with LGS require polytherapy. ${ }^{31}$ These results align very closely with a similar European expert opinion survey; however as second-line agents they recommended clobazam, ethosuximide, clonazepam and levetiracetam (Figure 1). ${ }^{32}$ Please note that zonisamide and felbamate are not currently approved for use in Canada but may be obtained by means of a Special Access Program through Health Canada, provided specific criteria are met. Overall, treatment of LGS is guided by empirical evidence, expert opinion, and individual clinical judgment. $^{31}$

\section{Valproic Acid}

Valproic acid is generally the first-line treatment of choice for patients with LGS. ${ }^{5}$ Its introduction predates the use of randomized controlled trials for LGS. In a 1982 study of 38 patients with "Lennox syndrome," the majority saw a $\geq 50 \%$ improvement. ${ }^{33}$ Valproic acid is most effective for the treatment of myoclonic, atypical absence, and atonic seizures ${ }^{5,34}$ However, valproic acid is a potent hepatic inhibitor. ${ }^{5}$ Liver toxicity is a rare but serious side effect, which occurs more often in those below the age of three and those receiving polytherapy. Tremor and weight gain have also been observed in patients taking valproic acid. ${ }^{35}$ Clinicians must be aware of the number of different interactions valproic acid has with other AEDs. ${ }^{5}$

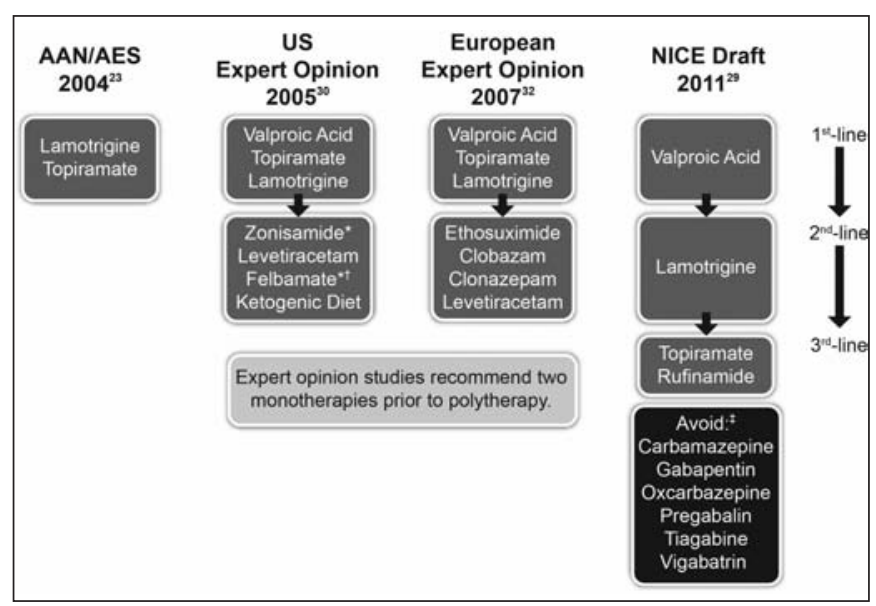

Figure 1: Overview of first-, second-, and third-line treatment options for the management of LGS, according to available guidelines and expert opinion publications. *Not approved for use in Canada. †NICE recommends felbamate be used only as a last resort due to the risk of toxicity. ${ }^{29} \neq$ Recommendation to avoid these seizure medications in patients with LGS since they may exacerbate certain types of seizures. 


\section{Lamotrigine}

In a randomized controlled trial of lamotrigine in 169 patients with LGS, $33 \%$ of those on lamotrigine versus $16 \%$ of those in the control group experienced a $50 \%$ or greater reduction in seizures. ${ }^{36}$ However, serious rash resulting in hospitalization occurred in $0.3 \%$ of adults and $1.0 \%$ of pediatric patients taking lamotrigine in clinical trials; and there is also a risk of StevensJohnson syndrome. ${ }^{37}$ The risk of rash can be reduced with a cautious titration schedule, over a period of up to eight weeks. ${ }^{38}$ This delay to maintenance dose can especially be an issue for patients with LGS experiencing multiple daily seizures. ${ }^{5}$ Moreover, the addition of carbamazepine, phenytoin or phenobarbital reduces the half-life of lamotrigine, while valproic acid can greatly increase plasma levels; therefore coadministration of these agents requires careful titration of lamotrigine to ensure therapeutic levels are reached and adverse events (AEs) are minimized..$^{31}$

\section{Topiramate}

In a randomized controlled trial in 98 patients with LGS a $50 \%$ or greater reduction in major seizures was seen in $33 \%$ of the topiramate group versus $8 \%$ of the control group..$^{39}$ The most common AEs were central nervous system-related, including language difficulties, behavioural problems and nervousness. Topiramate has not been associated with any life-threatening systemic AEs. ${ }^{38}$

\section{Felbamate}

Felbamate is also used for the treatment of LGS. Its efficacy was demonstrated in a double-blind, randomized controlled clinical trial. ${ }^{40}$ In this study, the felbamate-treated group showed a $34 \%$ decrease from baseline in the frequency of atonic seizures, compared with a $9 \%$ decrease in the placebo group $(\mathrm{p}=0.01)$. Felbamate-treated patients also had a significantly greater decrease in total seizure frequency compared with placebo patients (19 and 4\%, respectively; $\mathrm{p}=0.002$ ). Due to its severe adverse events in adults, felbamate can only be obtained through the Special Access Program. However, the treatment is well tolerated in children. ${ }^{41}$

\section{Levetiracetam}

Despite being suggested as a second-line agent in the aforementioned surveys of European and American expert opinion panels, ${ }^{30,32}$ no randomized controlled trial of levetiracetam in LGS has been conducted. In a retrospective case series of six patients with LGS, four patients experienced freedom from myoclonic seizures, four patients experienced freedom from generalized tonic-clonic seizures, and two patients experienced a $50 \%$ or greater reduction in atonic seizures. ${ }^{42}$ The most significant side effect was irritability.

\section{Benzodiazepines}

A randomized controlled trial of the benzodiazepine clobazam has been conducted in 68 patients with LGS ${ }^{43}$ Eightythree percent of patients in the high-dose group and thirty-eight percent of patients in the low-dose group experienced a $50 \%$ or greater reduction in seizures. Somnolence, lethargy and sedation were the most common AEs. Benzodiazepines have been shown to exacerbate the frequency of seizures in children with LGS, in particular tonic seizures. ${ }^{31}$ Intravenous injections of benzodiazepines are also known to cause status epilepticus in some patients. ${ }^{44}$

\section{Ethosuximide}

While no trials have been conducted to date, ethosuximide has been used in LGS to treat atypical absence seizures with some success. ${ }^{38}$

\section{Medications which can exacerbate seizures}

As mentioned above, some medications can actually exacerbate certain types of seizures and should either be avoided or used with caution in patients with LGS who experience multiple seizure types. ${ }^{45-49}$ According to the most recent NICE guidelines, carbamazepine, gabapentin, oxcarbazepine, pregabalin, tiagabine and vigabatrin should be avoided in these patients based on guideline development group feedback. ${ }^{29}$

\section{Non-pharmacological Treatment Options \\ Ketogenic Diet}

The ketogenic diet is a high fat, low carbohydrate, low protein diet used to control seizures. ${ }^{5,38}$ Its mechanism of action is still poorly understood..$^{5}$ The diet requires a high degree of compliance. ${ }^{38}$ In a study of 17 patients with LGS all patients experienced a $50 \%$ or greater reduction in atonic and myoclonic seizure frequency within the first five days of initiating the diet. ${ }^{50}$ An increase in alertness has also been reported. ${ }^{5}$ Potential AEs include renal stones, hyperuricemia, acidosis, metabolic derangements, ${ }^{38}$ vomiting, constipation, hunger, and lack of energy. 5

\section{Vagus Nerve Simulation (VNS)}

Although its exact mechanism of action remains to be defined, VNS has shown to be a useful alternative for patients with refractory epilepsy that cannot benefit from curative surgery. ${ }^{51}$ In a multi-centre retrospective study of 50 patients with LGS who underwent VNS, the median reduction in total seizures was $57.9 \%$ at six months. Other studies have also reported an increase in alertness in $76.7 \%$ of participants. ${ }^{52}$ The improvement seen as a result of VNS is gradual, with continual improvement over time. ${ }^{53}$ Common AEs include voice alteration, coughing during stimulation ${ }^{51}$ and drooling. ${ }^{52}$ No lifethreatening AEs have been reported ${ }^{38}$ and the procedure is reversible. ${ }^{53}$ In a prospective study of all patients with epilepsy treated with VNS at Hôpital Sainte-Justine over a four-year period (18\% of whom had LGS), weekly increase of stimulation intensity resulted in a significant improvement in every child with LGS within three months. ${ }^{54}$ In another study, specifically in patients with LGS $(\mathrm{N}=14)$, two experienced a $50 \%$ or greater reduction in drop attacks and one patient experienced a decrease in drop attack intensity or duration. ${ }^{55}$

\section{Corpus Callosotomy}

Corpus callosotomy is another surgical palliative procedure in which the corpus callosum connecting the two hemispheres of the brain is either partially or completely sectioned in order to 
Table 1: Summary of baseline demographics of participants in the double-blind trial and open-label extension. ${ }^{4,58}$

\begin{tabular}{c|c|c|c}
\hline Characteristic & $\begin{array}{c}\text { Rufinamide } \\
(\mathbf{n = 7 4 )}\end{array}$ & $\begin{array}{c}\text { Placebo } \\
(\mathbf{n = 6 4 )}\end{array}$ & $\begin{array}{c}\text { Extension } \\
(\mathbf{n = 1 2 4})\end{array}$ \\
\hline Male & $62.2 \%$ & $62.5 \%$ & $59.7 \%$ \\
\hline Female & $37.8 \%$ & $37.5 \%$ & $40.3 \%$ \\
\hline $4-<12$ years of age & $41.9 \%$ & $51.6 \%$ & $46 \%$ \\
\hline $12-<17$ years of age & $25.7 \%$ & $26.6 \%$ & $25.8 \%$ \\
\hline$\geq 17$ years of age & $32.4 \%$ & $21.9 \%$ & $28.2 \%$ \\
\hline $\begin{array}{l}\text { Duration of LGS } \\
\text { median (range) years }\end{array}$ & $7.9(0.1-32.7)$ & $7.5(0.1-34.1)$ & $9.8(0.07-34)$ \\
\hline
\end{tabular}

LGS: Lennox-Gastaut syndrome

reduce the frequency of drop attacks. ${ }^{12}$ In a study of 14 patients with LGS who underwent a total corpus callosotomy, $64.3 \%$ experienced a $\geq 50 \%$ reduction in seizure frequency at the 12 month follow-up. ${ }^{53}$ Corpus callosotomy has been reported to produce dramatic improvement immediately after surgery, which declines with time. Potential AEs include: decreased speech output, hemiparesis, gait disturbance, and disconnection syndrome. ${ }^{5}$ Corpus callosotomy is a relatively invasive and irreversible procedure typically used after failure of multiple medications..$^{30,32,53}$

\section{Rufinamide, A New Therapeutic Option}

Rufinamide is a drug which was recently approved for the adjunctive treatment of seizures associated with LGS in the US, Europe and Canada. ${ }^{12}$ Rufinamide is a triazole derivative and a wide spectrum anticonvulsant, structurally dissimilar to all other seizure medications. ${ }^{56}$ Like many other seizure medications its mechanism of action in humans has yet to be fully elucidated; however rufinamide's effect is thought to be via its ability to prolong the inactive state of sodium channels. This likely limits the ability of action potentials to fire at a high frequency. Rufinamide does not interact with GABA, adenosine, NMDA, monoaminergic, cholinergic, or excitatory amino acid binding sites. Rufinamide is an oral agent indicated in Canada for the adjunctive treatment of seizures associated with LGS in children four years-of-age and older and adults. ${ }^{57}$

A randomized controlled trial of rufinamide was conducted in patients with LGS to assess its efficacy and safety as adjunctive therapy. ${ }^{4,58}$

The baseline characteristics of the rufinamide and placebo groups were similar, with a greater number of male patients in each group (Table 1). ${ }^{4}$ The study consisted of a 28 -day baseline period, a 14-day titration period, and a 70-day maintenance period. The trial was followed by an open-label extension study (Figure 2) ${ }^{58}$ The three primary efficacy variables of the study were percent change in total seizure frequency per 28 days, percent change in tonic-atonic seizure frequency per 28 days and seizure severity rating. ${ }^{4}$ The three secondary efficacy variables were percentage of patients with a $50 \%$ or greater reduction in seizure frequency (responder rate), percent change in seizure frequency per 28 days for each seizure type other than tonicatonic, and parental global evaluation of condition. All comparisons were made between the 28-day baseline period and the double-blind phase.

The target dose of approximately $45 \mathrm{mg} / \mathrm{kg} /$ day was achieved by $87.8 \%(n=65)$ of the patients in the rufinamide group. ${ }^{4}$ The majority of patients reached the target dose within approximately seven days. A significant difference between rufinamide-treated patients and placebo-treated patients was found for all three primary efficacy variables. The median seizure frequency per 28 days was reduced $32.7 \%$ from baseline in the rufinamide group, compared to an $11.7 \%$ reduction in the placebo group. In terms of tonic-atonic seizure frequency per 28 days, a $42.5 \%$ reduction in median seizure frequency from baseline was observed in the rufinamide group, compared to a $1.4 \%$ increase from baseline in the placebo group (Figure 3).

Rufinamide treatment also significantly reduced the frequency of absence, atypical absence and atonic seizures, while all other seizure types recorded showed trends to greater

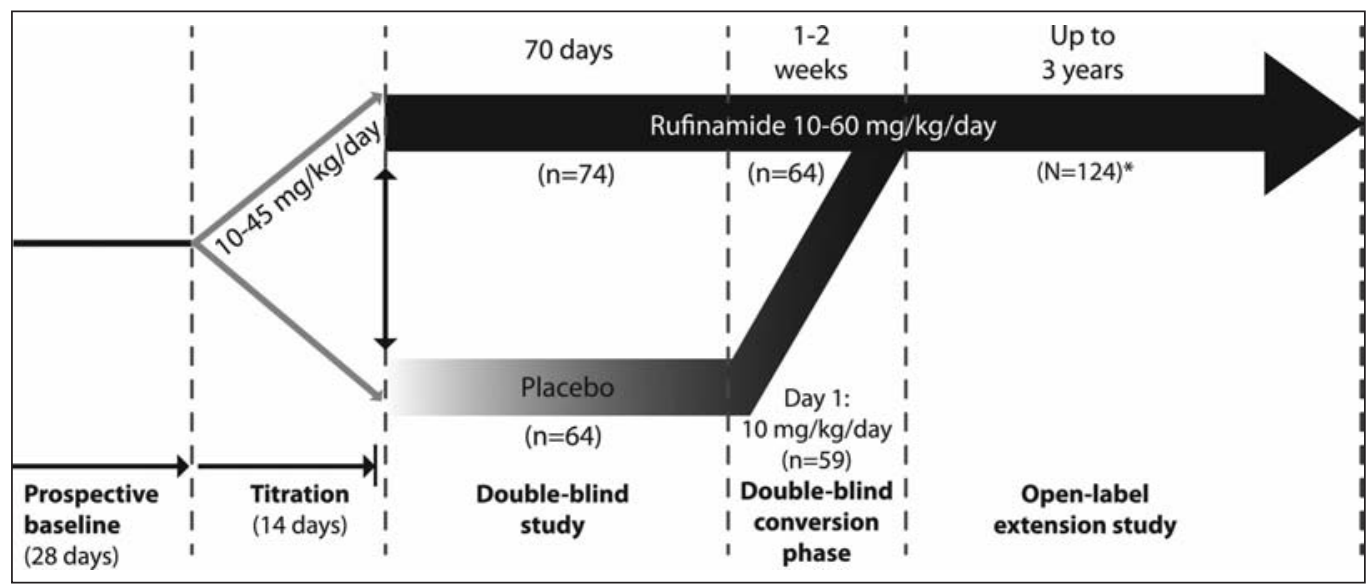

Figure 2: Rufinamide double-blind trial design with open-label extension study. 4,58*One patient bypassed doubleblind titration prior to extension phase. 


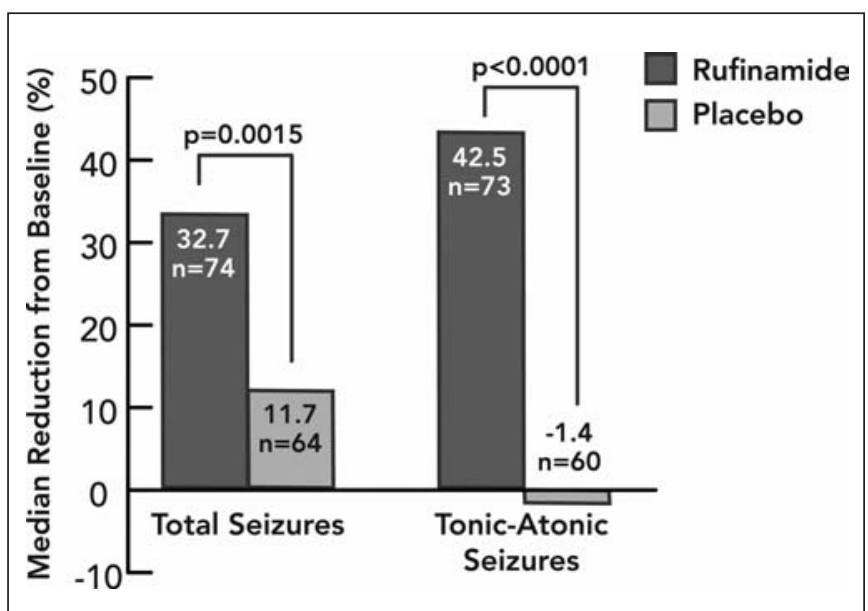

Figure 3: Change in total seizure frequency and tonic-atonic seizure frequency* per 28 days relative to baseline. $t^{4} *$ Tonic-atonic seizure includes both tonic and atonic seizures. fIncludes all intent-to-treat patients.

reductions with rufinamide compared to placebo group (Figure 4) ${ }^{4}$ Further, $53.4 \%$ of parents/guardians of rufinamide-treated patients versus $30.6 \%$ of parents/guardians of placebo-treated patients reported an improvement in seizure severity $(\mathrm{p}=0.0041)$. There was a greater percentage of responders in the rufinamidetreated group, compared to placebo, for both total seizures and tonic-atonic seizures, whether responders were defined as patients who experienced a $\geq 50$ or $\geq 75 \%$ reduction in seizure frequency (Figure 5). ${ }^{4}$ (Eisai Limited, data on file)

Following completion of the 12-week maintenance period of the randomized controlled trial, participants were given the option of continuing on in an open-label extension phase..$^{58}$ Of the 138 patients who were involved in the double-blind trial, 124 participated in the open-label extension. Since patients

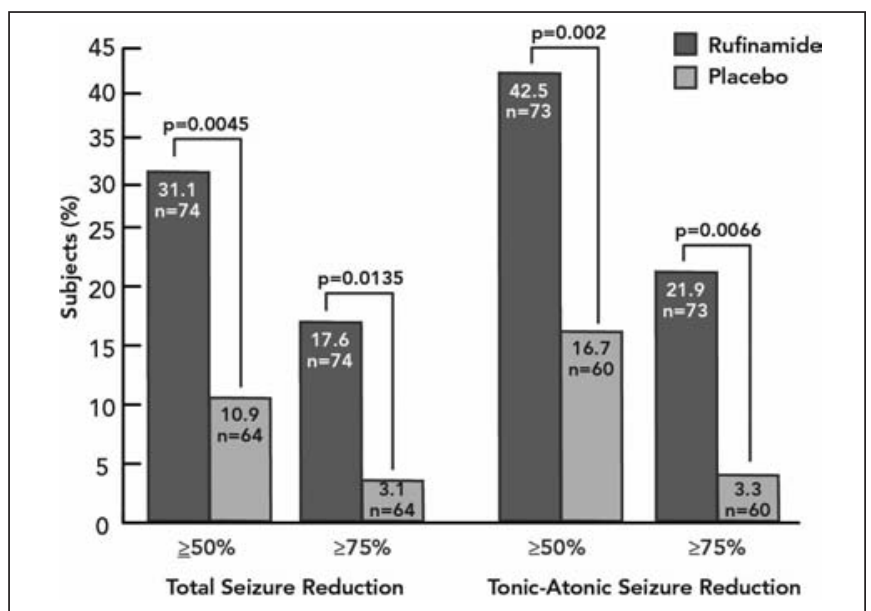

Figure 5: Responder rates for patients in the rufinamide and placebo groups (greater than or equal to $50 \%$ or $75 \%$ reduction in frequency of total seizures or tonic-atonic seizures). ${ }^{4}$

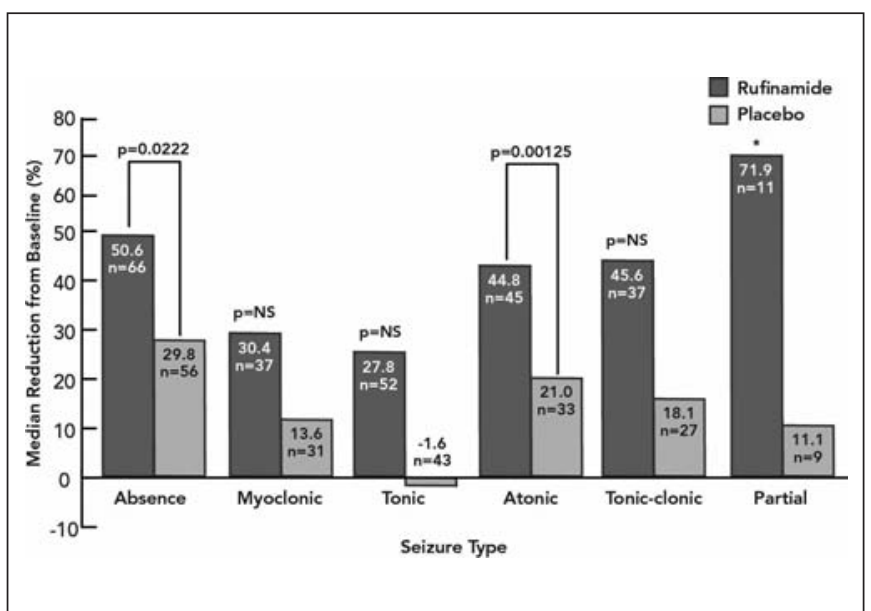

Figure 4: Percent change in frequency of various seizure types per 28 days relative to baseline. ${ }^{*}$ No p-value reported because partial seizures occurred in less than $20 \%$ of patients. NS: not significant.

previously on placebo were switched to rufinamide, all 124 patients participating in the extension study were on rufinamide. During the initial nine months of rufinamide treatment a progressive reduction in seizure frequency was observed, which reached roughly a 50 to $70 \%$ decrease in total seizure frequency and remained stable for up to three years. ${ }^{58} \mathrm{~A}$ reduction in median seizure frequency compared to baseline was seen at every time point over the three year duration of the study (Figure 6A). Changes in tonic-atonic seizure frequency showed a similar progressive reduction, reaching approximately a 60 to $80 \%$ decrease in frequency and remaining stable for up to three years (Figure 6B). A reduction in median tonic-atonic seizure frequency was seen at every time point over the three year duration of the study. In the final 12 months of treatment $47.9 \%$ of patients achieved a $50 \%$ or greater reduction in total seizures and $41.0 \%$ of patients achieved a $50 \%$ or greater reduction in tonic-atonic seizures. Within the final six months of treatment 11 patients on rufinamide (9.4\%) achieved freedom from tonicatonic seizures, and two patients $(1.6 \%)$ were seizure free. Approximately $48 \%$ of patients reduced their daily dose of concomitant AEDs during their first six months of rufinamide treatment.

Somnolence and vomiting were the only AEs which were significantly more common in the rufinamide group than the placebo group. ${ }^{4}$ Six patients (out of 74) in the rufinamide group withdrew from the study due to AEs, these included: vomiting $(\mathrm{n}=3)$, somnolence $(\mathrm{n}=2)$, and rash $(\mathrm{n}=2)$. Serious AEs were rare, occurring at a rate of $2.7 \%$ in the rufinamide group and $3.1 \%$ in the placebo group. In the rufinamide group, diarrhea, upper respiratory tract infection and vomiting were reported in one patient and rash, fatigue and vomiting in another. In the placebo group one patient reported sinusitis and one reported "petit mal epilepsy." Cognitive/psychiatric AEs occurred at a lower rate in the rufinamide group than in the placebo group. In general AEs observed in the three year, open-label phase of the study were similar to those observed in the double-blind phase. ${ }^{4,58}$ No clinically significant changes in laboratory values, vital signs or 


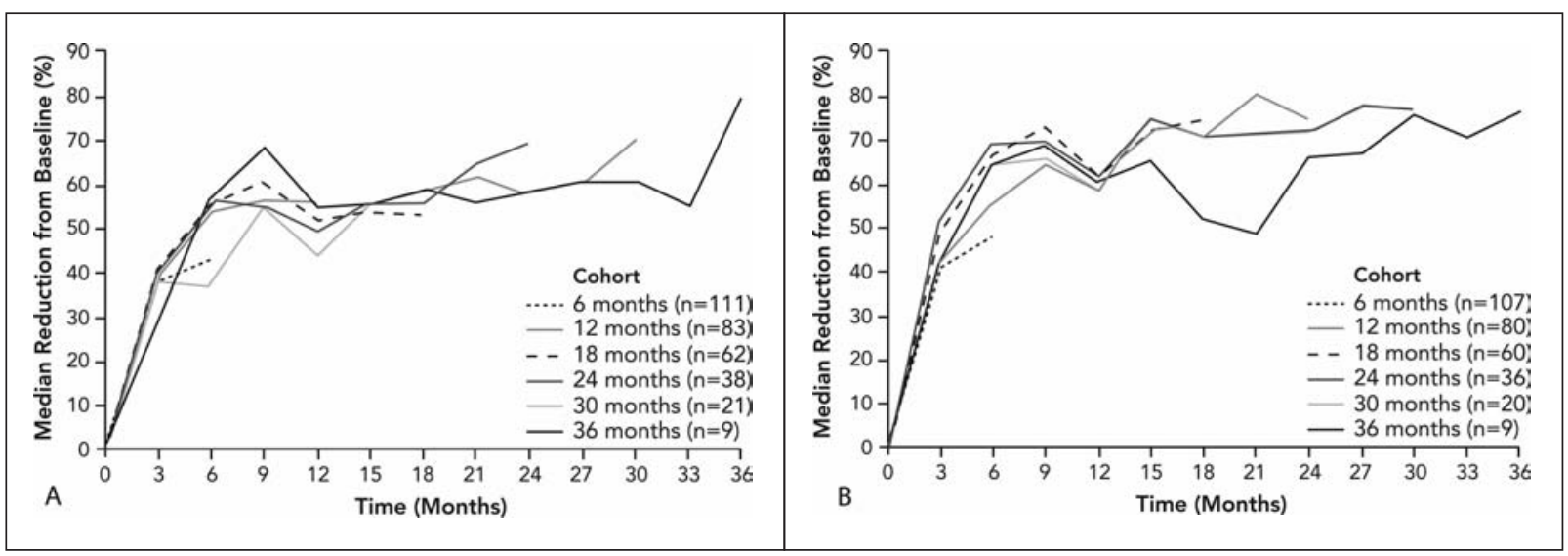

Figure 6: Median percent reduction from baseline in total A and tonic-atonic B seizure frequency over time by cohort of patients receiving rufinamide in the open-label extension study. ${ }^{58}$ (Note: patients on placebo in the double-blind trial were switched to rufinamide, therefore there is no comparator arm.)

electrocardiogram (ECG) were observed. Table 2 contains an overview of the safety data collected from the double-blind and extension phases of the pivotal trial.

Overall rufinamide has been administered to 1978 patients with epilepsy in clinical trials. ${ }^{59}$ When this data is pooled, the most common AEs (observed in more than ten percent of the population, with a greater incidence than placebo) were headache, dizziness, fatigue, somnolence and nausea. These side effects were usually mild or moderate in nature and transient. Only eight percent of participants in these studies discontinued rufinamide as a result of AEs.

Several post-marketing studies have been conducted in Germany/Austria, the US and Italy. ${ }^{60-62}$ While the Italian study examined rufinamide's use only in LGS, the American and the German/Austrian studies investigated the use of rufinamide in childhood onset refractory epilepsy. Rufinamide was well tolerated in the three post-marketing studies, which had followup periods of 18 (Germany/Austria), 21 (Italy) and 10 months (U.S.). Most of the AEs observed in these post-marketing studies were mild or moderate in intensity, transient (occurring early on in titration and subsiding in the maintenance period), or could be controlled by reducing dosage. The German/Austrian study reported that patients with LGS had one of the highest retention rates of the six epilepsy syndromes under study, at $51.6 \%$ (16 out of 31 patients) at 18 months. ${ }^{60}$ In patients with LGS the responder rates for total seizure frequency ( $\geq 50 \%$ reduction) were $35.5 \%, 38.4 \%$ and $60.5 \%$ (Germany/Austria, Italy and the US, respectively). ${ }^{60-62}$

In the double-blind trial and the open-label extension study concomitant AEDs used by more than ten percent of the

Table 2: Safety overview of rufinamide from the double-blind and open-label extension study. ${ }^{4,58}$ Most common AEs ( $\geq 10 \%$ of patients in any treatment group)

\begin{tabular}{|c|c|c|c|}
\hline & \multicolumn{2}{|c|}{ Double-blind Trial } & \multirow{2}{*}{$\begin{array}{c}\text { Open-label Study }^{*} \\
\text { Rufinamide }(\mathrm{N}=124) \%\end{array}$} \\
\hline & Rufinamide (n=74) \% & Placebo $(n=64) \%$ & \\
\hline Total AEs & 81.1 & 81.3 & 91.1 \\
\hline Somnolence & 24.3 & 12.5 & 21.0 \\
\hline Vomiting & 21.6 & 6.3 & 30.6 \\
\hline Pyrexia & 13.5 & 17.2 & 25.8 \\
\hline Nasopharyngitis & 9.5 & 3.1 & 17.7 \\
\hline Appetite decreased & 9.5 & 4.7 & 14.5 \\
\hline Fatigue & 9.5 & 7.8 & 13.7 \\
\hline Anorexia & 6.8 & 7.8 & 12.9 \\
\hline Headache & 6.8 & 4.7 & 10.5 \\
\hline Diarrhea & 5.4 & 10.9 & 17.7 \\
\hline Upper respiratory tract infection & 5.4 & 9.4 & 21.8 \\
\hline Constipation & 5.4 & 7.8 & 12.1 \\
\hline Aggression & 2.7 & 4.7 & 11.3 \\
\hline
\end{tabular}

*While a greater incidence of AEs was observed in the open-label study, this is thought to be a due to the longer duration of this study in comparison to the double-blind trial. In the open-label study rufinamide was administered for up to three years, in contrast to 12 weeks in the double-blind study. AE: adverse event. 
Table 3: Summary of drug-drug interactions of rufinamide with other AEDs.*57

\begin{tabular}{|c|c|c|}
\hline AED coadministered & $\begin{array}{l}\text { Influence of rufinamide on } \\
\text { AED concentration }\end{array}$ & Influence of AED on rufinamide concentration \\
\hline Carbamazepine & Decrease by $7 \%-13 \%$ & $\begin{array}{c}\text { Decrease by } 19 \%-26 \% \\
\text { (Dependent on dose of carbamazepine) }\end{array}$ \\
\hline Lamotrigine & Decrease by $7 \%-13 \%$ & No effect \\
\hline Phenobarbital & Increase by $8 \%-13 \%$ & $\begin{array}{c}\text { Decrease by } 25 \%-46 \% \\
\text { (Independent of dose or concentration of phenobarbital) }\end{array}$ \\
\hline Phenytoin & Increase by $7 \%-21 \%$ & $\begin{array}{c}\text { Decrease by } 25 \%-46 \%{ }^{8 \|} \\
\text { (Independent of dose or concentration of phenytoin) }\end{array}$ \\
\hline Topiramate & No effect & No effect \\
\hline Valproate & No effect & $\begin{array}{c}\text { Increase by }<16 \%-70 \% \\
\text { (Dependent on concentration of valproate) }\end{array}$ \\
\hline Primidone & Not investigated & $\begin{array}{c}\text { Decrease by } 25 \%-46 \% \\
\text { (Independent of dose or concentration of primidone) }\end{array}$ \\
\hline Benzodiazepines $^{\text {Tl }}$ & Not investigated & No effect \\
\hline
\end{tabular}

*Based on a population pharmacokinetic analysis. $†$ Predictions are based on rufinamide concentrations at the maximum recommended dose of rufinamide. $\$$ Maximum changes predicted to be in children and in patients who achieve significantly higher levels of rufinamide, as the effect of rufinamide on these AEDs is concentration-dependent. §Larger effects in children at high doses/concentrations of AEDs. II Phenobarbital, primidone, and phenytoin were treated as a single covariate (phenobarbital-type inducers) to examine the effect of these agents on rufinamide clearance. IAll compounds of the benzodiazepine class were pooled to examine for class effect on rufinamide clearance. AED: antiepileptic drug.

participants included valproic acid, lamotrigine, topiramate, clonazepam, carbamazepine, phenytoin, phenobarbital, and levetiracetam. ${ }^{4,58}$ Drug-drug interactions between rufinamide and other seizure medications are listed in Table $3 .{ }^{57}$ In the double-blind trial and open-label extension, rufinamide was taken concomitantly with the four agents whose concentration has been shown to be affected by rufinamide..$^{4,57,58}$ No dosage adjustments or impact on the efficacy or safety of rufinamide were reported in the double-blind or open-label extension phases of the pivotal trial., ${ }^{4}$ The only study to mention a dosage adjustment with concomitant use of rufinamide was the Italian post-marketing study. ${ }^{62}$ In patients less than $30 \mathrm{~kg}$, rufinamide was titrated up to a maximum dose of $600 \mathrm{mg}$ /day for those on concomitant valproic acid, as opposed to $1000 \mathrm{mg} /$ day. It is recommended that patients already on valproic acid start rufinamide at a dose lower than the recommended starting dose of $200 \mathrm{mg}$ /day in patients less than $30 \mathrm{~kg}$ and $400 \mathrm{mg} /$ day in those greater than or equal to $30 \mathrm{~kg} .{ }^{57}$ Patients stabilized on rufinamide should begin valproic acid therapy at a low dose, and titrate to a clinically effective dose.

In the double-blind trial the rufinamide dose was increased as quickly as $10 \mathrm{mg} / \mathrm{kg} /$ day every two days, over a period of seven days. ${ }^{4}$ The trial permitted a slower schedule over 14 days if tolerability issues arose. In the two post-marketing studies of rufinamide in which the titration schedule was reported, titration was performed at a slower rate. ${ }^{61,62}$ This may reflect the preference of physicians in the epilepsy community to titrate seizure medications slowly. For this reason, the recommended titration schedule for rufinamide in Canada is a $5 \mathrm{mg} / \mathrm{kg} /$ day increase every two weeks until satisfactory seizure control is reached (Table 4). ${ }^{57}$

\section{Prognosis Of LGS}

Lennox-Gastaut syndrome, in general, has a poor prognosis. Factors predictive of poorer outcomes include: history of West syndrome, cognitive impairment before onset of LGS,

Table 4: Starting dose and maximum recommended daily dose of rufinamide, according to patient weight. ${ }^{57}$

\begin{tabular}{|c|c|c|c|c|}
\hline Patient weight (kg) & $<30.0$ & $30.0-50.0$ & $50.1-70.0$ & $\geq 70.1$ \\
\hline Starting dose (mg/day) & 200 & \multicolumn{3}{|c|}{400} \\
\hline Titration Schedule & \multicolumn{4}{|c|}{$5 \mathrm{mg} / \mathrm{kg} /$ day every 2 weeks, after an evaluation of efficacy } \\
\hline $\begin{array}{c}\text { Maximum recommended } \\
\text { dose (mg/day) }\end{array}$ & 1300 & 1800 & 2400 & 3200 \\
\hline
\end{tabular}


symptomatic LGS, high frequency of status epilepticus, onset of first seizure before three years-of-age, and persistence of diffuse slowing of the background combined with generalized slow spike-and-wave pattern. ${ }^{7,11}$ It remains to be proven how much of the cognitive decline is due to the underlying pathology versus the ongoing frequent seizures. It would be interesting to study whether early use of newer seizure medications could limit the cognitive dysfunction; such an impact has been observed in Dravet syndrome. Less severe cognitive decline has been reported in more recent longitudinal studies of patients with Dravet syndrome and may be attributable to newer medication. ${ }^{63}$

\section{CONCLUSION}

Rufinamide adds to our current arsenal of treatment in LGS which includes: valproic acid, lamotrigine, topiramate, levetiracetam, ethosuximide, clobazam, clonazepam, zonisamide, felbamate, the ketogenic diet, vagus nerve stimulation, and corpus callosotomy. Rufinamide is effective and well-tolerated in both randomized controlled trials and observational studies of patients with LGS. The reduction in drop attacks and tonic-clonic seizures observed with rufinamide is clinically relevant for patients, may lead to a better quality of life and could modify long-term outcome. The medication was approved in Canada in 2011 for the adjunctive treatment of seizures associated with LGS in those aged four years and older, and gives Canadian physicians another medication to treat the devastating seizures of LGS.

\section{ACKNOWLEDGEMENTS}

The authors of this Review participated in a meeting of Canadian LGS experts that was funded by Eisai Canada, and the discussions at this meeting form the basis of this Review. This Review was prepared by the authors using a summary of the meeting produced by a medical writer (Meducom Health Inc.) who was funded by Eisai Canada. Eisai Canada has not contributed to the content of this Review. The authors thank C. Orth at Meducom Health Inc. who provided editorial assistance.

\section{REFERENCES}

1. Niedermeyer E. The Lennox-Gastaut syndrome: a severe type of childhood epilepsy. Dtsch Z Nervenheilkd. 1969;195(4):263-82.

2. Proposal for revised classification of epilepsies and epileptic syndromes. Commission on classification and terminology of the International League Against Epilepsy. Epilepsia. 1989;30(4): 389-99.

3. Berg AT, Berkovic SF, Brodie MJ, et al. Revised terminology and concepts for organization of seizures and epilepsies: report of the ILAE Commission on Classification and Terminology, 20052009. Epilepsia. 2010;51(4):676-85.

4. Glauser T, Kluger G, Sachdeo R, Krauss G, Perdomo C, Arroyo S. Rufinamide for generalized seizures associated with LennoxGastaut syndrome. Neurology. 2008;70(21):1950-8.

5. Michoulas A, Farrell K. Medical management of Lennox-Gastaut syndrome. CNS Drugs. 2010;24(5):363-74.

6. Rantala H, Putkonen T. Occurrence, outcome, and prognostic factors of infantile spasms and Lennox-Gastaut syndrome. Epilepsia. 1999;40(3):286-9.

7. Markand ON. Lennox-Gastaut syndrome (childhood epileptic encephalopathy). J Clin Neurophysiol. 2003;20(6):426-41.

8. Camfield P. Definition and natural history of Lennox-Gastaut syndrome. Epilepsia. 2011;52(Suppl. 5):3-9.

9. Camfield CS, Camfield PR, Gordon K, Wirrell E, Dooley JM. Incidence of epilepsy in childhood and adolescence: a population based study in Nova Scotia from 1977 to 1985. Epilepsia. 1996;37:19-23.

10. Trevathan E, Murphy CC, Yeargin-Allsopp M. Prevalence and descriptive epidemiology of Lennox-Gastaut syndrome among Atlanta children. Epilepsia. 1997;38(12):1283-8.

11. Dulac O, Nguyen T. The Lennox-Gastaut syndrome. Epilepsia. 1993;34:S7-S17.

12. Arzimanoglou A, French J, Blume WT, et al. Lennox-Gastaut syndrome: a consensus approach on diagnosis, assessment, management, and trial methodology. Lancet Neurol. 2009;8(1): 82-93.

13. Deekollu D, Besag FMC, Aylett SE. Seizure-related injuries in a group of young people with epilepsy wearing protective helmets: incidence, types and circumstances. Seizure. 2005;14 (5):347-53.

14. Gallop K, Wild D, Nixon A, Verdian L, Cramer JA. Impact of Lennox-Gastaut syndrome (LGS) on health-related quality of life (HRQL) of patients and caregivers: literature review. Seizure. 2009;18(8):554-8.

15. Ferlazzo E, Nikaronova M, Italiano D, et al. Lennox-Gastaut syndrome in adulthood: clinical and EEG features. Epilepsy Res. 2010;89(2-3):271-7.

16. Beaumanoir A. The Lennox-Gastaut syndrome: a personal study. Electroencephalogr Clin Neurophysiol Suppl. 1982;(35):85-99.

17. Chevrie JJ, Aicardi J. Childhood epileptic encephalopathy with slow spike-wave. A statistical study of 80 cases. Epilepsia. 1972; 13(2)259-71.

18. Berg AT, Scheffer IE. New concepts in classification of the epilepsies: entering the 21st century. Epilepsia. 2011;52(6): 1058-62.

19. Harkin LA, McMahon JM, Iona X, et al. The spectrum of SCNIArelated infantile epileptic encephalopathies. Brain. 2007;130: 843-52.

20. Selmer KK, Lund C, Brandal K, Undlien DE, Brodtkorb E. SCN1A mutation screening in adult patients with Lennox-Gastaut syndrome features. Epilepsia. 2009;50:555-7.

21. Miller IO, Sotero de Menezes MA. SCN1A-related seizure disorders. In: Pagon RA, Bird TD, Dolan CR, Stephens K, editors. GeneReviews. Seattle (WA): University of Washington; 1993.

22. You SJ, Kim HD, Kang H. Factors influencing the evolution of West syndrome to Lennox-Gastaut syndrome. Pediatr Neurol. 2009;41(2):111-3.

23. French J, Kanner AM, Bautista J, et al. Efficacy and tolerability of the new antiepileptic drugs, II: Treatment of refractory epilepsy. Report of the TTA and QSS subcommittees of the American Academy of Neurology and the American Epilepsy Society. Epilepsia. 2004;45(5):410-23.

24. van Rijckevorsel K. Treatment of Lennox-Gastaut syndrome: overview and recent findings. Neuropsychiatr Dis Treat. 2008;4 (6):1001-19.

25. Goldsmith I, Zupanc M, Buchhalter J. Long-term seizure outcome in 74 patients with Lennox-Gastaut syndrome: effects of incorporating MRI head imaging in defining the cryptogenic subgroup. Epilepsia. 2000;41(4):395-9.

26. Yagi K. Evolution of Lennox-Gastaut syndrome: a long-term longitudinal study. Epilepsia. 1996;37:48-51.

27. Gallop K, Wild D, Verdian L, et al. Lennox-Gastaut syndrome (LGS): development of conceptual models of health-related quality of life (HRQL) for caregivers and children. Seizure. 2010;19(1):23-30.

28. French JA, Kanner AM, Bautista J, et al. Efficacy and tolerability of the new antiepileptic drugs I: treatment of new onset epilepsy - report of the therapeutics and technology assessment subcommittee and quality standards subcommittee of the American Academy of Neurology and the American Epilepsy Society. Neurology. 2004;62(8):1252-60.

29. National Institute for Clinical Excellence (NICE) [Internet]. The epilepsies - the diagnosis and management of the epilepsies in adults and children in primary and secondary care. NICE partial update of clinical guidelines. [Updated 2011 January; cited 2011 October]. Available from: http://www.nice.org.uk.libaccess.lib. mcmaster.ca/nicemedia/live/12108/52447/52447.pdf. 
30. Wheless J, Clarke D, Carpenter D. Treatment of pediatric epilepsy: expert opinion, 2005. J Child Neurol. 2005;20:S1-S56.

31. Montouris G. Rational approach to treatment options for LennoxGastaut syndrome. Epilepsia. 2011;52(Suppl. 5):10-20.

32. Wheless JW, Clarke DF, Arzimanoglou A, Carpenter D. Treatment of pediatric epilepsy: European expert opinion, 2007. Epileptic Disord. 2007:9(4):353-412.

33. Covanis A, Gupta A, Jeavons P. Sodium valproate - monotherapy and polytherapy. Epilepsia. 1982;23(6):693-720.

34. Jeavons P, Clark J, Maheshwari M. Treatment of generalized epilepsies of childhood and adolescence with sodium valproate (Epilim). Dev Med Child Neurol. 1977;19(1):9-25.

35. Yu PM, Zhu GX, Wu X, et al. A 6-month prospective study on efficacy safety and QOL profiles of extended-release formulation of valproate in patients with epilepsy. Seizure. 2011; 20(1):23-6.

36. Motte J, Trevathan E, Arvidsson J, et al. For generalized seizures associated with the Lennox-Gastaut syndrome. N Engl J Med. 1997;337(25): 1807-12.

37. Messenheimer J. Rash in adult and pediatric patients treated with lamotrigine. Can J Neurol Sci. 1998;25(4):S14-8.

38. Schmidt D, Bourgeois B. A risk-benefit assessment of therapies for Lennox-Gastaut syndrome. Drug Saf. 2000;22(6):467-77.

39. Sachdeo R, Glauser T, Ritter F, Reife R, Lim P, Pledger G. A double-blind, randomized trial of topiramate in Lennox-Gastaut syndrome. Neurology. 1999;52(9):1882-7.

40. The Felbamate Study Group in Lennox-Gastaut Syndrome. Efficacy of felbamate in childhood epileptic encephalopathy (Lennox-Gastaut syndrome). The Felbamate Study Group in Lennox-Gastaut Syndrome. N Engl J Med. 1993;328(1):29-33.

41. Carmant L, Holmes GL, Sawyer S, Rifai N, Anderson J, Mikati MAl. Efficacy of felbamate in therapy for partial epilepsy in children. J Pediatr. 1994;125(3):481-6.

42. de los Reyes E, Sharp G, Williams J, Hale S. Levetiracetam in the treatment of Lennox-Gastaut syndrome. Pediatr Neurol. 2004;30 (4):254-6

43. Conry JA, Ng Y, Paolicchi JM, et al. Clobazam in the treatment of Lennox-Gastaut syndrome. Epilepsia. 2009;50(5):1158-66.

44. Tassinari CA, Dravet C, Roger J, Cano JP, Gastaut H. Tonic status epilepticus precipitated by intravenous benzodiazepine in five patients with Lennox-Gastaut syndrome. Epilepsia. 1972;13(3): 421-35.

45. Gelisse P, Genton T, Kuate C, Pesenti A, Baldy-Moulinier M, Crespel A. Worsening of seizures by oxcarbazepine in juvenile idiopathic generalized epilepsies. Epilepsia. 2004;45(10): $1282-6$

46. Genton P, Gelisse P, Thomas P, Dravet C. Do carbamazepine and phenytoin aggravate juvenile myoclonic epilepsy? Neurology. 2000;55(8):1106-9.

47. Asconape J, Diedrich A, DellaBadia J. Myoclonus associated with the use of gabapentin. Epilepsia. 2000;41(4):479-81
48. Schapel G, Chadwick D. Tiagabine and non-convulsive status epilepticus. Seizure. 1996;5(2):153-6.

49. Lortie A, Chiron C, Mumford J, Dulac O. The potential for increasing seizure frequency, relapse, and appearance of new seizure types with vigabatrin. Neurology. 1993;43(11):S24-7.

50. Freeman J, Vining E. Seizures decrease rapidly after fasting preliminary studies of the ketogenic diet. Arch Pediatr Adolesc Med. 1999;153(9):946-9.

51. Frost $\mathbf{M}$, Gates $\mathbf{J}$, Helmers $\mathrm{S}$, et al. Vagus nerve stimulation in children with refractory seizures associated with LennoxGastaut syndrome. Epilepsia. 2001;42(9):1148-52.

52. Kostov K, Kostov H, Tauboll E. Long-term vagus nerve stimulation in the treatment of Lennox-Gastaut syndrome. Epilepsy Behav. 2009;16(2):321-4.

53. You SJ, Kang H, Ko T, et al. Comparison of corpus callosotomy and vagus nerve stimulation in children with Lennox-Gastaut syndrome. Brain Dev. 2008;30(3):195-9.

54. Rossignol E, Lortie A, Thomas T, et al. Vagus nerve stimulation in pediatric epileptic syndromes. Seizure. 2009;18(1):34-7.

55. Zamponi N, Passamonti C, Cesaroni E, Trignani R, Rychlicki F. Effectiveness of vagal nerve stimulation (VNS) in patients with drop-attacks and different epileptic syndromes. Seizure. 2011;20(6):468-74.

56. Bialer M, Johannessen S, Kupferberg H, Levy R, Loiseau P, Perucca E. Progress report on new antiepileptic drugs: a summary of the fifth EILAT conference (EILAT V). Epilepsy Res. 2001;43(1):11-58.

57. Banzel® (rufinamide) [product monograph]. Mississauga, ON: Eisai Limited; June 20, 2011.

58. Kluger G, Glauser T, Krauss G, Seeruthun R, Perdomo C, Arroyo $\mathrm{S}$. Adjunctive rufinamide in Lennox-Gastaut syndrome: a longterm, open-label extension study. Acta Neurol Scand. 2010;122 (3):202-8

59. Wisniewski CS. Rufinamide: a new antiepileptic medication for the treatment of seizures associated with Lennox-Gastaut syndrome. Ann Pharmacother. 2010;44(4):658-67.

60. Kluger G, Haberlandt E, Kurlemann G, et al. First European longterm experience with the orphan drug rufinamide in childhoodonset refractory epilepsy. Epilepsy Behav. 2010;17(4):546-8.

61. Vendrame M, Loddenkemper T, Gooty VD, et al. Experience with rufinamide in a pediatric population: A single center's experience. Pediatr Neurol. 2010;43(3):155-8.

62. Coppola G, Grosso S, Franzoni E, et al. Rufinamide in children and adults with Lennox-Gastaut syndrome: first Italian multicenter experience. Seizure. 2010;19(9):587-91.

63. Chieffo D, Battaglia D, Lettori D, et al. Neuropsychological development in children with Dravet syndrome. Epilepsy Res. 2011;95(1-2):86-93. 